\title{
A complexidade da disponibilização e acesso a dados governamentais na Web
}

Fábio Mosso Moreira

\begin{abstract}
Graduação em Administração de Empresas pela Universidade Estadual Paulista Campus de Tupã Mestrado pelo Programa de Pós Graduação em Ciência da Informação, na Linha de Informação e Tecnologia, da Universidade Estadual Paulista - Faculdade de Filosofia e Ciências de Marília.Membro do Grupo de Pesquisa - Novas Tecnologias em Informação
\end{abstract}

Ricardo César Gonçalves Sant'Ana

Professor assistente da Universidade Estadual Paulista Júlio de Mesquita Filho, Campus de Tupã .Professor do Programa de Pós-Graduação em Ciência da Informação da Universidade Estadual Paulista, Campus de Marília.Doutorado em Ciência da Informação pela Universidade Estadual Paulista Júlio de Mesquita Filho

Maria José Vicentini Jorente

Doutora pelo Programa de Pós-Graduação em Ciência da Informação da Universidade Estadual Paulista Júlio de Mesquita Filho (Unesp).Especialista em Design de Produto.Professora Assistente- da Universidade Estadual Paulista Júlio de Mesquita Filho, Faculdade de Filosofia e Ciências - FFC - Campus de Marília, Departamento de Ciência da Informação.Editora de conteúdo do periódico científico BRAJIS

http://dx.doi.org/10.1590/1981-5344/2540 
A Web pode ser considerada um recurso promissor para 0 compartilhamento de informações públicas. O crescente interesse dos governos em viabilizar a implantação de seus portais a fim de obter maior eficiência e transparência em suas ações comunicacionais impacta na distribuição de dados governamentais. Pelo fato de o dado não transmitir uma mensagem ou representar algum conhecimento, características complexas podem ser observadas no processo de distribuição de dados. Esta pesquisa tem como objetivo descrever a relação entre propriedades da Complexidade com características identificadas no processo de disponibilização e acesso a dados governamentais na Web, apontando como estas propriedades podem se manifestar no fluxo informacional. A metodologia foi baseada em pesquisa qualitativa, por meio de um estudo exploratório realizado a partir de um levantamento bibliográfico que identificou conceitos sobre a Teoria dos Sistemas Complexos e aspectos envolvidos no compartilhamento de dados governamentais na Web.

Palavras-chave: Dados Governamentais; Sistemas Complexos; Complexidade; Web.

\section{Complexity of provision and access of government data in the Web}

The Web can be regarded as a promising resource for the sharing of public information. The distribution of government data has been impacted by the governments' crescent interest in making possible the implementation of web portals to improve efficiency and transparency of their communication actions. Complex features can be observed in the government data distribution process, since data itself does not transmit a message or represent knowledge. This research aims at describing the relation between properties of the Complexity with identified features in the delivery and access processes to government data on the Web, pointing out how these properties can be manifested in the informational flow. By 
an exploratory study and a literature review, the methodology was based on a qualitative research that identified concepts of Theory of Complex Systems and aspects involved in the sharing of government data on the Web.

Keywords: Governmental Data; Complex System; Complexity; Web.

Recebido em 03.11.2015 Aceito em 23.05.2016

\section{Introdução}

As novas Tecnologias de Informação e Comunicação (TIC) e a evolução dos dispositivos digitais proporcionaram meios para viabilizar maior interação e comunicação entre a administração pública e indivíduos e grupos da sociedade, tanto para os cidadãos que buscam por informações governamentais para o processo de participação democrática quanto para o Governo que deve fornecer mecanismos de relação com serviços públicos mais eficientes e transparentes.

Neste contexto, Sant'Ana (2009) ressalta a importância dos governos em disponibilizarem informações sobre suas ações, fornecendo dados sobre: planos e programas governamentais; orçamentos, arrecadação e fontes de receita; licitações, contratos e seus aditivos; e dados sobre funcionários municipais e município. $O$ acesso a este conteúdo pode permitir maior acompanhamento das ações do poder público, gerando maior confiança no Estado, assim como no processo político e governamental.

A responsabilidade das administrações públicas em disponibilizar informações sobre suas ações foi intensificada com a promulgação da Lei Federal no 12.527, de 2011. A Lei de Acesso à Informação (LAI) adicionou novas obrigações às instituições públicas, reforçando o dever do Estado em garantir o Direito de acesso à informação de forma transparente, clara e em linguagem de fácil compreensão, dispondo também sobre a obrigação da divulgação de informações em sítios oficiais da rede mundial de computadores (BRASIL. Presidência da República, 2011).

A Web têm se mostrado um recurso promissor para 0 compartilhamento de informações governamentais. Sant'Ana (2009) destaca o crescente interesse das administrações públicas em viabilizar a implantação de seus portais, seja por força da obrigatoriedade legal disposta pela LAI, seja pela necessidade de se utilizar desta ferramenta de divulgação a fim de obter maior eficiência e transparência em suas ações de comunicação.

$O$ acesso às informações e dados governamentais pode ser considerado elemento chave para a construção de uma participação 
cidadã ativa e de relação mais transparente entre administração pública e sociedade (SANT'ANA; RODRIGUES, 2013). Neste sentido, torna-se fundamental a ampla distribuição de dados pelas instituições públicas a fim de que se estimulem demandas por parte dos cidadãos.

No processo de compartilhamento de dados deve-se considerar que este tipo de informação possui algumas particularidades, como o fato de que um dado por si só não transmitir uma mensagem ou representar algum conhecimento. A disponibilização e acesso a dados envolve um processo que compreende características complexas que podem ser mais bem compreendidas com base na Teoria dos Sistemas Complexos, considerando que a complexidade interna deste sistema está relacionada à sua estrutura e funcionalidade.

O sistema que envolve a disponibilização e acesso a dados governamentais na Web pode ser associado à definição de Wagensberg (2004) na qual o autor define sistemas complexos como sendo sistemas compostos de uma estrutura em rede, formada por nervuras ramificadas como um sistema nervoso, e que pode representar um objeto real ou abstrato. Estes sistemas podem ser compostos de nervuras tecnológicas, ou seja, um emaranhado de tecnologias compatíveis ou não, que aumenta sua dimensão em virtude das inter-relações que coexistem em cada nível de compatibilidade, resultando em um formato de rede (LOPEZ; MONTIEL, 2006).

Considerando que a estrutura e as funcionalidades envolvidas no compartilhamento de dados governamentais na Web podem ser entendidas, sob uma perspectiva sistêmica, como um sistema tecnológico complexo, esta pesquisa tem como objetivo descrever a relação entre propriedades da Complexidade com características envolvidas no processo de disponibilização e acesso a dados governamentais na Web, apontando como estas propriedades complexas podem manifestar-se neste fluxo informacional.

\section{Metodologia}

A metodologia adotada está pautada na análise qualitativa, de caráter exploratório, na qual se realizou um levantamento bibliográfico a fim de obter informações sobre o compartilhamento de dados governamentais na Web, e levantar conceitos sobre o tema da Complexidade e Teoria dos Sistemas Complexos. Com as informações obtidas foi possível identificar características envolvidas no processo de disponibilização e acesso a dados governamentais na Web, e delinear intersecções com propriedades da Complexidade, também definidas por meio de pesquisa teórica.

Os resultados obtidos foram sistematizados por meio da elaboração de quadros ilustrativos para representar as relações entre propriedades da Complexidade e características envolvidas no processo de disponibilização e acesso a dados governamentais na Web. Considera-se que a 
metodologia utilizada nesta pesquisa pode ser aplicada também por estudos que busquem analisar a distribuição de outros tipos de dados, como geográficos, demográficos, climáticos, econômicos, buscando obter perspectivas sistêmicas acerca do processo de disponibilização e acesso a dados em ambiente digital.

\section{Características da disponibilização e acesso a dados governamentais na Web}

A disponibilização e acesso à informação pública trazem possibilidades ao cidadão de participar e controlar as ações do Governo. No estado atual, em que impera a cultura do segredo, o Governo deve entender que o cidadão é titular da informação pública, mas muitas vezes confunde-se o acesso a uma informação como um favor que o agente público faz àquele cidadão (GALLO, 2012).

Segundo Lopes e Sant'Ana (2013) a Web têm promovido grandes avanços na esfera social, sendo um deles proporcionar meios para a distribuição de dados governamentais, contribuindo assim com a ampliação da transparência pública e participação democrática do cidadão. No Brasil, uma das primeiras iniciativas a utilizar a Web para o compartilhamento de dados do Governo ocorreu em 1994, com o Portal da Transparência da Controladoria-Geral da União, "[...] trazendo informações sobre a aplicação de recursos do governo federal, abrangendo transferências e aplicações diretas" (NETO; CRUZ; VIEIRA, 2006 , p. 137). Com esta iniciativa, o país buscou incorporar os preceitos de Governo Eletrônico, assumindo a Web como tecnologias promissoras na comunicação de conteúdos públicos e promoção do acompanhamento democrático de modo proativo.

O Governo Eletrônico envolve questões técnicas (como a utilização dos recursos tecnológicos) e organizacionais (como o tratamento e efetividade da administração pública). Os objetivos perseguidos são: transparência, acessibilidade, prestação de contas, serviços públicos eficientes, políticas públicas e envolvimento dos usuários. Ao incorporar os preceitos do Governo Eletrônico, as agências governamentais estão buscando eficiência e melhoria na qualidade dos dados disponibilizados (ANDERSEN; HENRIKISEN, 2006).

No contexto das questões técnicas do Governo Eletrônico, Sant'Ana (2009) debate a utilização de TIC por parte do Governo no gerenciamento de dados, ressaltando que o uso das tecnologias pode ser classificado em: interno (auxiliando nos processos internos da gestão e administração da coisa pública) e externo (facilitando a relação entre a organização pública com entidades externas, como empresas, cidadãos ou grupo de cidadãos e outras organizações públicas).

Com relação à utilização de TIC para uso Externo, os órgãos governamentais podem adotar dois modelos para o desenvolvimento de mecanismos promotores de transparência na disponibilização dos dados e informações em seus portais: a forma ativa (tornando disponíveis conjuntos de dados para livre acesso à população) e a forma passiva (oferecendo mecanismos para receber solicitações dos cidadãos para 
acesso a conjunto de dados ainda não disponíveis) (RODRIGUES; SANT'ANA, 2013).

Os dados possuem comportamentos distintos aos de uma informação quando inseridos no fluxo de um processo de recuperação. Segundo Rijsbergen (1979) na recuperação de dados busca-se por uma correspondência exata à necessidade, enquanto na recuperação da informação espera-se uma correspondência aproximada (a melhor correspondência possível); na recuperação de dados a inferência utilizada é a dedução lógica, enquanto na recuperação da informação é possível realizar uma inferência indutiva (possui graus de certeza ou incerteza); na recuperação de dados a linguagem utilizada é uma linguagem artificial restrita por sintaxes específicas, enquanto na recuperação da informação utiliza-se de uma linguagem natural que pode ser expressa apenas com as especificações necessárias; ainda sobre a linguagem de consulta utilizada, na recuperação de dados a linguagem, por ser amarrada a uma sintaxe, possui maior sensibilidade ao erro, enquanto na recuperação da informação, pequenos erros podem ser contornados.

Uma informação pode ser compreendida como um conjunto finito de dados dotados de semântica e que tem sua significação ligada ao contexto do agente que a interpreta, em meio a fatores como: tempo, contexto, forma de transmissão e suporte utilizado (SANTOS; SANT'ANA, 2002). Esta pesquisa adota como definição para dados a apresentada por Santos e Sant'Ana (2002), na qual os autores referem-se ao termo dado como um "elemento básico, formado por signo ou conjunto finito de signos que não contém, intrinsicamente, um componente semântico, mas somente elementos sintáticos". Esta natureza particular dos dados faz com que, na busca por conjuntos de dados governamentais na Web, sejam encontrados diversos problemas que apresentam-se como fatores de desmotivação no momento da consulta às bases disponíveis. Muitas vezes o acesso parcial, superficial e de difícil compreensão acaba inviabilizando possíveis análises e interpretações dos conjuntos de dados (LOPES; SANT'ANA, 2013).

O processo de compartilhar dados na Web é uma atividade que envolve fatores complexos. Com a intenção de resolver problemas encontrados na distribuição de dados públicos, no ano de 2011, o Governo brasileiro firmou um compromisso com o Open Government Partnership, do qual o país é um dos estados fundadores e colidera a parceria com os EUA. Para cumprimento dessa parceria, os sítios oficiais do Governo que tornam acessíveis seus conjuntos de dados devem atender aos oito princípios de dados abertos, utilizando as TIC para ampliar o acesso às fontes disponíveis e permitir que cidadãos tenham capacidade de recuperar dados que lhes sejam de interesse e atendam suas necessidades (RODRIGUES; SANT'ANA, 2012).

Segundo o Open Government Data (2007), os dados são considerados abertos se seguirem os seguintes princípios: serem dados completos, não se restringindo a limitação de segurança, privacidade ou privilégio. Todos os dados devem ser disponibilizados; serem dados 
primários, ou seja, dados colhidos na fonte, com nível mais fino de granularidade, não de forma agregada ou modificada; serem dados pontuais, disponibilizados tão rapidamente quanto necessário, preservando o valor dos dados; serem dados acessíveis, estando disponíveis para a mais ampla gama de usuários para as mais diversas finalidades; serem dados processáveis por máquinas; ter acesso nãodiscriminatório, ou seja, disponíveis a qualquer pessoa; serem dados nãoproprietários, estando disponíveis em qualquer formato sobre qual nenhuma entidade tem controle absoluto.

O Governo pode aumentar as possibilidades, para os cidadãos, de acesso a dados governamentais atendendo aos oito princípios de dados abertos, evitando assim, problemas comuns encontrados nesse processo, como o acesso parcial, superficial e de difícil compreensão dos dados. Contudo, ao observar o cenário atual da distribuição de dados governamentais, entende-se que nem todos os princípios estão sendo atendidos, pois a maioria dos portais governamentais ainda publicam dados sobre suas ações em formato fechado. Este tipo de publicação caracteriza-se pelo fato de que o Governo é quem decide o que e como será visto, tratando seus portais como ferramentas de promoção de sua imagem em vez de proporcionar infraestrutura técnica adequada para publicar os dados de forma aberta (VAZ; RIBEIRO; MATHEUS, 2010).

Para Robinson et al. (2009), na maioria das vezes os portais governamentais são utilizados para fins de propaganda política, contendo assim muitos elementos informacionais que dificultam a localização das fontes de dados disponibilizadas, em vez de fornecer uma interface simples de acesso e reuso dos dados. Além da grande quantidade de elementos, é uma característica frequente em muitos portais governamentais fornecer inúmeros ícones com links que direcionam para outras fontes de informação, o que pode indicar que o usuário não irá encontrar a informação desejada internamente àquele ambiente informacional (ANDERSEN; HENRIKISEN, 2006).

Sant'Ana e Rodrigues (2013) ressaltam que a disponibilização de dados governamentais não é realizada de forma centralizada, ou seja, cada órgão do Governo deve elaborar, em seus sítios oficiais, mecanismos para o atendimento dos aspectos legais referentes às transparências Ativa e Passiva, resultando em características heterogêneas de recursos informacionais e de conjuntos de dados pulverizados por diferentes sítios. Uma das formas de atender à transparência Ativa é fornecer opções de consultas aos dados disponibilizados, sendo que um mesmo conjunto de dados pode apresentar diversas possibilidades de mapeamento de seus atributos, levando a diferentes modelos e técnicas para visualização, e permitindo apresentar múltiplas visualizações a partir de um mesmo atributo selecionado (LOPES; SANT'ANA, 2013).

Segundo Vaz, Ribeiro e Matheus (2010), alguns portais governamentais já disponibilizam consultas personalizadas nas quais os interessados podem acessar as bases de forma interativa, configurando as consultas de forma que produzam o cruzamento de dados específicos ou 
parametrizando filtros em busca de informações detalhadas. Contudo, o Governo deve ir além de oferecer mecanismos de visualização de dados, ofertando também serviços como buscas avançadas, análise automatizada de conteúdo, e cruzamento com outras fontes de dados de forma simultânea (ROBINSON et al., 2009).

Muitas das informações disponíveis em portais governamentais estão "trancadas" em arquivos digitais, como imagens de documentos impressos, que não são facilmente reutilizáveis. Para Robinson et al. (2009) fornecer dados reutilizáveis reduz o custo prático do reuso, adaptação e inovação. A redução deste custo pode fazer com que emerjam, mais facilmente, iniciativas de terceiros no fornecimento de mecanismos exclusivos para recuperação dos dados governamentais por públicos específicos, visto que para o Governo é preferível fornecer portais como núcleo de sua responsabilidade em vez de viabilizar estruturas tecnológicas de compartilhamento de dados.

O fornecimento de dados reutilizáveis implica, necessariamente, na disponibilização de dados primários, que são dados de granularidade fina, e que não passaram por transformações ou manipulações, como somas, agrupamentos, análises ou qualquer outro tipo de processamento. A disponibilização de dados primários é um dos fatores-chave de sucesso na construção de alternativas para a disponibilização e acesso a dados governamentais na Web, evitando eventuais falhas de interpretação e ainda permitindo caminhos variados de análise em função do alto grau de liberdade de combinações e possíveis processamentos que um dado primário oferece (RODRIGUES; SANT'ANA, 2013).

$\mathrm{Na}$ disponibilização e acesso a dados governamentais um aspecto que também adiciona complexidade ao processo é o problema da privacidade. Em algumas situações típicas, os usuários, para interagir com um serviço online, devem fornecer significante quantidade de dados sensíveis, sendo que cada serviço do governo tem sua própria política de privacidade que envolve o uso, armazenamento e divulgação dos dados (MEDJAHED et al., 2003).

Muitas das características encontradas na distribuição de dados governamentais na Web envolvem fatores complexos que podem ser compreendidos sob a perspectiva da Teoria dos Sistemas Complexos. Para tanto, na seção seguinte é conceituada esta Teoria, com o intuito de identificar propriedades da Complexidade para serem relacionadas às características envolvidas no processo de disponibilização e acesso a dados governamentais na Web.

\section{Teoria dos sistemas complexos}

O nível atual das novas tecnologias informacionais está mudando radicalmente a forma de coletar e processar a informação, passando de uma abordagem monolítica para uma abordagem de colaboração em rede. A atual distribuição de dados encontra desafios específicos, como: integração, fusão, reconstrução espaço-temporal e validação. Com isso, muitos conjuntos de dados disponíveis na Web encontram-se incompletos, 
mal organizados e subqualificados (BOURGINE; CHAVALARIAS; PERRIER, 2009).

A Web pode ser entendida como um sistema infológico, que possibilita 0 acesso a elementos informacionais de diferentes tipos e estruturas, como conhecimentos, dados, imagens, ideias, fantasias, abstrações e crenças (BURGIN; SIMON, 1997). A existência de um alto volume de conteúdos informacionais distribuídos em formas distintas, compartilhados através de uma estrutura em rede, resulta em fatores que influenciam no estado de complexidade desse sistema.

O comportamento de economias nacionais, o cérebro humano e o ecossistema da floresta Amazônica são bons exemplos de sistemas complexos (CASTI, 2001). Segundo Abraham (2002), a história dos Sistemas Complexos por si só já é complexa, sendo concebida a partir de conexões e interações entre a Teoria Geral dos Sistemas, a Cibernética e a Teoria dos Sistemas Dinâmicos.

Embasados principalmente pela Teoria Geral dos Sistemas, que acrescenta uma visão holística ao estudo, os teóricos da Complexidade contemporânea focalizam a forma pelas qual as interações locais dos atores de um sistema o levam a um sistema global e ao modo como estas interações agem para manter e aumentar a complexidade do sistema (WARREN; FRANKLIN; STREETER, 1998).

Sistema Complexo pode ser definido como sendo qualquer sistema composto por um grande número de entidades heterogêneas, no qual as interações locais entre suas entidades criam múltiplos níveis de estrutura e organização coletiva (BOURGINE; CHAVALARIAS; PERRIER, 2009).

Souza e Buckeridge (2004, p.408) definem Sistemas Complexos como sendo sistemas "compostos de muitos elementos e/ou subsistemas diferentes interagindo espacialmente e temporalmente de forma nãolinear, gerando padrões emergentes que são observáveis apenas em escalas maiores".

Os Sistemas Complexos são estruturalmente e funcionalmente complexos. A complexidade estrutural se manifesta na variedade de elementos e conexões, e a complexidade funcional se manifesta através da variedade de funções e comportamentos (BURGIN; SIMON, 1997).

Para Morin (1977), a complexidade em um sistema ocorre entre o estado de ordem e desordem, e envolve a ideia de interação (que une os elementos), transformação (que pode organizar ou dispersar os elementos) e organização (que nasce das interações e transformações entre os elementos).

Serva, Dias e Alperstedt (2010), ao tratarem sobre Sistemas Complexos, dão destaque ao conceito da autonomia relacionada à reação dos elementos, e substanciam o estado de complexidade ressaltando a ideia de organização, auto-organização, ordem, desordem e evento. Os autores também ressaltam que a complexidade do sistema consiste na contínua autoprodução, auto-organização e autonomia dos elementos.

Para Casti (2001), o termo complexo pode ser usado para descrever pessoas e/ou objetos, concretos ou abstratos, constituídos de muitos 
componentes em interação, os quais possuem comportamento e estrutura difíceis de serem compreendidos. Segundo o autor, para se estudar sistemas complexos é necessário considerar algumas propriedades que os diferem de um sistema simples, como: a instabilidade (muitas possibilidades de comportamento), irredutibilidade (um todo unificado que não pode ser estudado em partes), adaptabilidade (presença de agentes inteligentes que tomam decisões com base em informações incompletas) e emergência. Estas propriedades, entre outras que também foram identificadas por meio das definições apresentadas nesta seção, são descritas detalhadamente na próxima seção.

\section{Propriedades da complexidade}

Com base nos conceitos levantados sobre a Teoria dos Sistemas Complexos foi possível estabelecer, baseado nas definições observadas, um conjunto com oito propriedades que podem ser consideradas fatores do estado de complexidade de um sistema. Estas propriedades estão sistematizadas no Quadro 1, no qual é possível observar que das oito propriedades definidas, cinco delas são abordadas por mais de um autor em comum, como: volume, heterogeneidade, interação, auto-organização e emergência. Outras três propriedades foram abordadas por um autor específico, como: instabilidade, irredutibilidade e adaptabilidade. Para cada propriedade presente no Quadro 1, são apresentados excertos das definições e conceitos levantados sobre a Teoria dos Sistemas Complexos (expostos na seção anterior) agrupados por cada um dos autores consultados que serviram de embasamento.

O volume pode ser considerado a primeira propriedade do estado de complexidade de um sistema complexo. Esta propriedade foi definida tendo como embasamento a descrição de autores que ressaltaram a presença de grande quantidade de elementos em um sistema complexo atuando continuamente em diferentes subsistemas. O volume está relacionado diretamente com a quantidade de entidades e suas ocupações em níveis distintos do sistema, sendo esta uma propriedade que pode variar em escala e nível ao longo do tempo.

A heterogeneidade foi definida como uma propriedade da complexidade por ser uma das características principais dos agentes pertencentes a um sistema complexo. Esta propriedade abrange condições como uniformidade, dessemelhança, formatos desiguais, distintos e irregulares, que resultam em uma composição variada de elementos presentes no sistema.

A interação é uma importante propriedade da complexidade por ser a ação responsável por conectar o alto volume de elementos heterogêneos por meio de conexões que podem ocorrer entre duas ou mais entidades simultaneamente. A interação permite que os elementos do sistema constituam grupos e desempenhem comportamentos que geram outros estímulos para que a ação seja recíproca. Sem interação entre as entidades, os sistemas complexos tendem a perder energia e se extinguir. 
Quadro 1 - Propriedades da Complexidade

\begin{tabular}{|c|c|c|c|c|c|c|}
\hline Propriedades & $\begin{array}{c}\text { BOURGINE, } \\
\text { CHAVALARIAS } \\
\text { \& PERRIER } \\
(2009) \\
\end{array}$ & $\begin{array}{c}\text { SOUZA \& } \\
\text { BUCKERIDGE } \\
(2004)\end{array}$ & $\begin{array}{c}\text { BURGIN \& } \\
\text { SIMON (1997) }\end{array}$ & MORIN (1977) & $\begin{array}{c}\text { SILVA, DIAS } \\
\& \\
\text { ALPERSTEDT } \\
(2010) \\
\end{array}$ & CASTI (2001) \\
\hline Volume & $\begin{array}{l}\text { Grande } \\
\text { número de } \\
\text { entidades }\end{array}$ & $\begin{array}{c}\text { Muitos } \\
\text { elementos e } \\
\text { subsistemas }\end{array}$ & & & & $\begin{array}{c}\text { Muitos } \\
\text { componentes }\end{array}$ \\
\hline Heterogeneidade & $\begin{array}{c}\text { Entidades } \\
\text { heterogêneas }\end{array}$ & $\begin{array}{c}\text { Elementos e } \\
\text { subsistemas } \\
\text { diferentes }\end{array}$ & $\begin{array}{l}\text { Variedade de } \\
\text { elementos e } \\
\text { conexões }\end{array}$ & & & \\
\hline Interação & $\begin{array}{l}\text { Interações } \\
\text { locais entre } \\
\text { entidades }\end{array}$ & $\begin{array}{c}\text { Interagindo } \\
\text { espacialmente } \\
e \\
\text { temporalmente }\end{array}$ & $\begin{array}{c}\text { Elementos e } \\
\text { conexões, } \\
\text { funções e } \\
\text { comportamentos } \\
\end{array}$ & $\begin{array}{l}\text { Interações e } \\
\text { transformações }\end{array}$ & & $\begin{array}{l}\text { Componentes } \\
\text { em interação }\end{array}$ \\
\hline $\begin{array}{l}\text { Auto- } \\
\text { organização }\end{array}$ & & & & $\begin{array}{c}\text { Organização } \\
\text { que nasce das } \\
\text { interações e } \\
\text { transformações } \\
\text { em meio ao } \\
\text { estado de } \\
\text { ordem e } \\
\text { desordem } \\
\end{array}$ & $\begin{array}{c}\text { A } \\
\text { complexidade } \\
\text { consiste na } \\
\text { contínua } \\
\text { autoprodução, } \\
\text { auto- } \\
\text { organização e } \\
\text { autonomia } \\
\end{array}$ & \\
\hline Emergência & & $\begin{array}{l}\text { Gerando } \\
\text { padrões } \\
\text { emergentes }\end{array}$ & & & & $\begin{array}{c}\text { Emergência de } \\
\text { padrões de } \\
\text { comportamento } \\
\text { que não } \\
\text { podem ser } \\
\text { previstos } \\
\end{array}$ \\
\hline Instabilidade & & & & & & $\begin{array}{c}\text { Muitas } \\
\text { possibilidades } \\
\text { e modos de } \\
\text { comportamento }\end{array}$ \\
\hline Irredutibilidade & & & & & & $\begin{array}{c}\text { Não pode ser } \\
\text { estudado em } \\
\text { partes, } \\
\text { unificado }\end{array}$ \\
\hline Adaptabilidade & & & & & & $\begin{array}{c}\text { Agentes } \\
\text { inteligentes } \\
\text { que tomam } \\
\text { decisões com } \\
\text { base em } \\
\text { informações } \\
\text { parciais e } \\
\text { incompletas }\end{array}$ \\
\hline
\end{tabular}

Fonte: Dados da pesquisa.

A interatividade entre os elementos do sistema também permite que as entidades se estruturem através de um processo de auto-organização, que ocorre em meio ao estado de ordem e desordem, proporcionando contínua adaptabilidade aos elementos do sistema. Esta propriedade está diretamente relacionada com a autonomia do sistema e reação de seus elementos frente aos estímulos, atuando de forma coordenada e independente no modo como configuram suas estruturas.

Associada ao processo de auto-organização, a emergência é definida como uma propriedade da complexidade por ser o fenômeno responsável pelo surgimento dos padrões e leis que fazem com que sejam configuradas as estruturas e funções do sistema. $O$ ato de emergir consiste na formação de padrões complexos a partir da multiplicidade de interações coletivas entre os elementos, resultando em modelos de comportamento que não podem ser facilmente previstos. 
A auto-organização e a emergência são responsáveis pelo alto grau de adaptabilidade dos elementos de um sistema complexo, que podem tomar decisões e agir com base em informações parciais e incompletas. As entidades do sistema são capazes de mudar suas regras de decisões baseadas nas partes de informações adquiridas, fazendo com que o sistema e seus elementos possam aprender sobre o ambiente em que estão inseridos, e mudar flexivelmente seu padrão de comportamento conforme os estímulos recebidos.

A adaptabilidade faz com que os agentes dos sistemas complexos possam ter inúmeros modos possíveis de comportamento, alternando entre estes modos como resultado de pequenas alterações em alguns dos fatores que o regem. As mudanças entre esses modos são frequentes, caracterizando um alto grau de instabilidade nas condições do sistema, que oscila tanto na questão estrutural quanto nos aspectos funcionais, sem firmar-se em uma condição única.

Todas as propriedades descritas anteriormente atuam como fatores do estado de complexidade de um sistema, agindo como um todo de forma unificada. A irredutibilidade é a propriedade da complexidade referente à natureza única dos sistemas complexos que não podem ser compreendidos pela desintegração das partes que os compõem, e nem pela análise de suas partes isoladamente. O comportamento do sistema é determinado pela composição entre todas as partes, sendo que qualquer desintegração pode destruir seus aspectos de individualidade.

As propriedades volume e heterogeneidade manifestam-se principalmente no âmbito estrutural dos sistemas complexos. No âmbito funcional, as propriedades interação, auto-organização e emergência configuram os comportamentos e atividades locais no sistema. Estas propriedades, atuando funcionalmente no âmbito estrutural do sistema, resultam na adaptabilidade, propriedade na qual os elementos assumem independentemente novas configurações de forma ativa; na instabilidade, como resultado da contínua oscilação entre os modos de comportamento do sistema; e na irredutibilidade, propriedade relacionada à dependência da conexão entre todas as partes do sistema como um todo e de forma unificada.

\section{Resultados e discussões}

Para traçar uma relação entre propriedades da complexidade e características do processo de disponibilização e acesso a dados governamentais na Web, considerou-se uma estrutura composta por três níveis distintos presentes no sistema: o nível mais amplo, no qual se encontram as relações de acesso dos usuários aos portais governamentais na Web; o nível médio, referente aos elementos que constituem o conteúdo presente em cada um destes portais, como os conjuntos de dados, mídias, documentos públicos; e o nível mais rígido, relacionado especificamente à estrutura dos conjuntos de dados, como forma e atributos dos registros disponíveis em cada uma das bases.

No Quadro 2 estão relacionadas algumas das propriedades da complexidade definidas na seção anterior associadas com características 
presentes no processo de disponibilização e acesso a dados governamentais na Web, apontando intersecções encontradas entre estes dois contextos e descrevendo como as propriedades da complexidade podem manifestar-se neste sistema.

Quadro 2 - Propriedades da complexidade no processo de disponibilização e acesso a dados governamentais na Web

\begin{tabular}{c|l}
$\begin{array}{c}\text { Propriedade da } \\
\text { Complexidade }\end{array}$ & \multicolumn{1}{|c}{ Características do processo de disponibilização e acesso a dados } \\
Vovernamentais na Web
\end{tabular}

Fonte: Dados da pesquisa.

O volume é uma propriedade da complexidade que pode ser relacionada à descrição de Robinson et al. (2009), na qual o autor referese à presença de muitos elementos informacionais que dificultam a localização das fontes de dados nos portais governamentais. Considerando que os conjuntos de dados disponibilizados nos sítios constituem um tipo específico de elementos informacionais que compõem o conteúdo total do portal, e que os portais são, em sua maioria, utilizados para fins de propaganda política, verifica-se a presença de inúmeros outros tipos elementos informacionais distintos, distribuídos juntos às bases de dados disponibilizadas. Neste sentido, pode-se considerar que o volume manifesta-se no nível médio da estrutura deste sistema, no qual se encontram os elementos que compõem o conteúdo presente nestes portais (bases de dados, recursos publicitários, mídias). Destaca-se que 
esta propriedade pode manifestar-se também no nível mais amplo da estrutura deste sistema, devido à grande quantidade de portais governamentais acessíveis na Web, e no nível mais exíguo, onde cada base de dados compõe-se de inúmeros registros e atributos, verificando assim a manifestação desta propriedade nos três níveis considerados na pesquisa.

A heterogeneidade é uma propriedade da complexidade que pode ser relacionada à descrição de Sant'Ana e Rodrigues (2013), na qual os autores ressaltam a heterogeneidade de recursos informacionais e conjuntos de dados disponibilizados pelos sítios do Governo. Esta condição de uniformidade pode ser associada à descentralização no processo de disponibilização das informações públicas, onde cada órgão do Governo é responsável por elaborar, em seus sítios oficiais, mecanismos para o acesso aos conjuntos de dados. Desta forma, considera-se que a heterogeneidade manifesta-se no nível do acesso por parte dos usuários aos distintos portais governamentais na Web onde se encontram diferentes tipos de elementos informacionais e mecanismos disponíveis para acessar as bases. Sobre os mecanismos de consulta, verifica-se a heterogeneidade nas distintas tecnologias utilizadas por cada portal, sendo que em alguns sítios é possível encontrar aplicações avançadas que permitem realizar buscas avançadas e cruzamento de dados com bases externas, enquanto outros portais disponibilizam dados de forma "fechada", dificultando a reutilização dos dados.

A instabilidade é uma propriedade da complexidade que pode ser relacionada à descrição de Andersen e Henrikisen (2006), na qual os autores destacam como característica frequente em muitos portais governamentais o fornecimento de inúmeros ícones com links que levam quem está acessando o conteúdo para diferentes fontes de dados externas ao sítio, ao invés de direcionar para um ponto centralizador que agregue todas as consultas e conjuntos de dados disponíveis. Os inúmeros links que direcionam para as bases de dados externas costumam ser alterados frequentemente, quando não se encontram indisponíveis, gerando graus de inconsistência no nível do acesso aos conjuntos de dados e demais conteúdos informacionais, de forma que esta instabilidade pode prejudicar a obtenção da informação desejada e influenciar na credibilidade do portal.

A interação é uma propriedade da complexidade que pode ser associada à descrição de Vaz, Ribeiro e Matheus (2010), em que os autores destacam que alguns portais governamentais já disponibilizam mecanismos de consultas avançadas, onde usuários podem acessar bases de dados de forma interativa, parametrizando consultas de modo com que produzam o cruzamento específico dos dados. Desta forma, considera-se que a manifestação da interação pode ocorrer no nível do acesso aos portais e seu conteúdo, onde o interessado em obter uma informação pode configurar o mecanismo de consulta conforme suas necessidades e habilidades. 
A interação pode ser relacionada também com a descrição de Medjahed et al. (2003) destacando que em muitos casos o usuário para interagir com um serviço público online de disponibilização de dados deve fornecer significante quantidade de dados pessoais, levantando 0 problema da privacidade no processo de acesso a dados governamentais. Os autores destacam que cada portal do Governo pode ter sua própria política de privacidade envolvendo o uso, armazenamento e divulgação dos dados, caracterizando uma condição de heterogeneidade nas políticas de privacidade dos portais. Em ambos os casos, considera-se que as duas propriedades manifestam-se no nível do acesso aos portais e seu conteúdo: a interação durante o fornecimento de informações pessoais na utilização dos serviços; e a heterogeneidade nas distintas políticas de privacidade em que o usuário submete-se ao consultar o conteúdo.

Rodrigues e Santa'Ana (2013) destacam a importância da disponibilização de dados governamentais em sua forma primária. Dados primários (dados com alto grau de granularidade e baixo/nenhum grau de processamento) podem evitar falhas de interpretações e permitir seu reuso com alto grau de liberdade de combinações possíveis. A disponibilização de base de dados em sua forma primária pode permitir que tecnologias semânticas, como $S P A R Q L^{1}$, realizem consultas simultâneas em fontes distintas e de forma interligada, facilitando a combinação dos dados. Neste sentido, pode-se dizer que a propriedade interação pode manifestar-se também no nível médio da estrutura deste sistema, definido pelos cruzamentos entre distintas bases de dados internas e externas ao sítio.

\section{Considerações finais}

A pesquisa apresentou resultados da aplicação de uma análise qualitativa, com enfoque sistêmico, para entendimento dos aspectos envolvidos no processo de disponibilização e acesso a dados governamentais na Web, buscando levantar perspectivas baseadas nos conceitos da Teoria dos Sistemas Complexos, por meio da identificação de propriedades da Complexidade e definição de intersecções entre estas e os fatores atuantes no compartilhamento de dados governamentais em meio digital.

Com o estudo realizado foi possível estabelecer relações entre quatro propriedades da complexidade, das oito definidas nesta pesquisa, com as características da disponibilização e acesso a dados governamentais na Web. Baseado no levantamento de características envolvidas no compartilhamento de dados governamentais foi possível verificar que o volume, a heterogeneidade, a instabilidade, e a interação, são propriedades da complexidade que podem manifestar-se neste sistema de fluxo informacional.

\footnotetext{
1 SPARQL Protocol and RDF Query Language (SPARQL) é uma linguagem que permite consultas em uma base de dados específica ou entre diversas bases de dados ao mesmo tempo, tendo como resultado dados em forma de tabelas ou grafos RDF (WORLD WIDE WEB CONSORTIUM - W3C, 2013).
} 
Entender como fatores complexos atuam no processo de disponibilização e acesso a dados governamentais na Web, sob um viés filosófico na Ciência da Informação, pode contribuir com a área embasando outros estudos que busquem abordar aspectos comunicacionais entre Governo e Sociedade, gerando perspectivas qualitativas a respeito da questão que envolve o compartilhamento de dados governamentais.

\section{Referências}

ABRAHAM, H. R. The Genesis of Complexity. In: MONTUORI, A. Advances in systems theory, complexity, and the human sciences. Santa Cruz: Visual Math Institute, 2002. Disponível em: <http://www.ralphabraham.org/articles/MS\%23108.Complex/complex.pdf $>$. Acesso em: 29 jan. 2015.

ANDERSEN, K. V.; HENRIKISEN. H. Z. E-Government maturity models: extension of the layne and lee model. Government Information Quarterly, v. 23, n. 1,2006 . Disponível em: $<$ http://www.sciencedirect.com/science/article/pii/S0740624X05000973 >. Acesso em: 20 fev. 2015.

BOURGINE, P.; CHAVALARIAS, D.; PERRIER, E. The CSS roadmap for the science of complex systems. Paris: Assyst, 2009. Disponível em: <http://www.complexssociety.eu/texts/roadmaps/ASSYST roadmap2009 2.pdf>. Acesso em: 15 jan. 2015.

BRASIL. Presidência da República. Lei n. 12.527, de 18 de novembro de 2011. Regula o acesso a informações previsto no inciso XXXIII do art. 5o, no inciso II do § 30 do art. 37 e no § 20 do art. 216 da Constituição Federal; altera a Lei no 8.112, de 11 de dezembro de 1990; revoga a Lei no 11.111 , de 5 de maio de 2005, e dispositivos da Lei no 8.159 , de 8 de janeiro de 1991; e dá outras providências. Portal do Planalto, Brasília, DF, 18 nov. 2011. Disponível em: <http://www.planalto.gov.br/ccivil 03/ Ato20112014/2011/Lei/L12527.htm >. Acesso em: 31 abr. 2014.

BURGIN, M.; SIMON, I. Information, energy and evolution. Los Angeles: University of California, 1997. Disponível em: <http://cogprints.org/2359/1/Evolnf.pdf>. Acesso em: 28 jan. 2015.

CASTI, J. L. The simply complex. Santa Fe: Santa Fe Institute, 2001. Disponível em: <http://quantumcybernetics.org/UniWork/Papers of Interest files/basicscomplexCasti.pdf $>$. Acesso em: 15 jan. 2014. 
GALLO, F. Acesso à informação não é favor, é direito. O Estado de São Paulo, São Paulo, 22 jan. 2012. Disponível em: $<$ http://politica.estadao.com.br/noticias/eleicoes, acesso-a-informacao-nao-e-favor-edireito-imp-,825658 >. Acesso em: 28 jan. 2013.

LOPES, R. de C. C.; SANT'ANA, R. C. G. Percepção dos usuários sobre o processo de acesso a dados sobre saúde em sítios do Governo Federal. In: ENCONTRO NACIONAL DE PESQUISA EM CIÊNCIA DA INFORMAÇÃO ENANCIB, 14., 2013, Florianópolis. Anais..., Florianópolis: ANCIB, 2013. Disponível

em:

$<$ http://enancib2013.ufsc.br/index.php/enancib2013/XIVenancib/paper/viewFile/188/ 309>. Acesso em: 7 mar. 2015.

LOPEZ, M. P.; MONTIEL, M. La Tecnología en la complejidad del siglo XXI. In: SEMINARIO DEL CONSTRUCTIVISMO, 2005, 1., Maracaibo. Anais... Maracaibo: Universidad del Zulia, 2006. Disponível em: $<$ http://gte2.uib.es/edutec/sites/default/files/congresos/edutec07/aprobedutec07/docs 212.doc>. Acesso em: 9 ago. 2016.

MEDJAHED, B. et al. Infrastructure for E-Government Web Services. IEEE Computer Society, v. 7, n. 1, p. 58-65, 2003. Disponível em: $<$ http://ieeexplore.ieee.org/stamp/stamp.jsp?tp=\&arnumber=1167340 $>$. Acesso em: 20 fev. 2015.

MORIN, E. O método 1: da natureza da natureza. 2. ed. Porto Alegre: Sulina, 1977.

NETO, O. A. P.; CRUZ, F. da; VIEIRA, A. L. Transparência das contas públicas: um enfoque no uso da Internet como instrumento de publicidade na UFSC. Revista Contemporânea de Contabilidade, v. 1, n. 5, p. 135-146, $2006 . \quad$ Disponível em: $<$ https://periodicos.ufsc.br/index.php/contabilidade/article/view/1143>. Acesso em: 20 fev. 2015.

OPEN GOVERNMENT DATA. 8 principles of open government data. [S.I]: OpenGovData, $2007 . \quad$ Disponível em: <http://www.opengovdata.org/home/8principles >. Acesso em: 22 fev. 2015.

RIJSBERGEN, C. J. V. Information retrieval. 2. ed. Newton-MA: Butterworth-Heinemann, $1979 . \quad$ Disponível em: <http://dl.acm.org/citation.cfm?id=539927>. Acesso em: 9 ago. 2016.

ROBINSON, D. et al. Government Data and the Invisible Hand. Yale Journal of Law and Technology, v. 12, n. 1, 2009. Disponível em: <http://yjolt.org/government-data-and-invisible-hand>. Acesso em: 20 fev. 2015. 
RODRIGUES, F. de A.; SANTANA, R. C. G. Restrições tecnológicas e de acesso a dados disponíveis sobre destino de repasse financeiros federais para a saúde pública em ambientes informacionais digitais. In: ENCONTRO NACIONAL DE PESQUISA EM CIÊNCIA DA INFORMAÇÃO ENANCIB, 2012, 13., Rio de Janeiro-RJ. Anais... Rio de Janeiro: ANCIB, 2012. Disponível

em: <http://www.eventosecongressos.com.br/metodo/enancib2012/arearestrita/pdfs/194 35.pdf >. Acesso em: 7 mar. 2015.

RODRIGUES, F. A.; SANT'ANA, R. C. G. Uso de modelos de dados multidimensionais para a ampliação da transparência ativa. Liinc em Revista, v .9, n. 2, 2013. Disponível em: <http://revista.ibict.br/liinc/index.php/liinc/article/view/599>. Acesso em: 7 mar. 2015.

SANT'ANA, R. C. G. Tecnologia e gestão pública municipal: mensuração da interação com a sociedade. São Paulo: Cultura Acadêmica Editora; UNESP, 2009. Disponível em: <http://www.cairu.br/biblioteca/arquivos/Tecnologia/Tecnologia e Gestao Publica Municipal.pdf >. Acesso em: 7 mar. 2015.

SANTANA, R. C. G.; RODRIGUES, F. A. Visualização de afinidades entre parlamentares mediante dados de votações no Senado Brasileiro. Informação \& Sociedade (UFPB. Online), v. 23, n. 1, p. 49-59, 2013. Disponível em: <http://www.ies.ufpb.br/ojs/index.php/ies/article/viewFile/12903/9264>. Acesso em: 7 mar. 2015.

SANTOS, P. L. A. da C.; SANT'ANA, R. C. G. Transferência da informação: análise para valoração de unidades de conhecimento. DataGramaZero, v. 3, n. 2, 2002. Disponível em: <http://www.dgz.org.br/abr02/Art 02.htm>. Acesso em: 7 mar. 2015.

SERVA, M.; DIAS, T.; ALPERSTEDT, G. D. Paradigma da complexidade e teoria das organizações: uma reflexão epistemológica. Revista de Administração de Empresas - RAE, v. 50, n. 3, p. 276-287, 2010. Disponível em: <http://rae.fgv.br/rae/vol50-num3-2010/paradigma-complexidadeteoria-organizacoes-reflexao-epistemologica>. Acesso em: 7 mar. 2015.

SOUZA, G. M.; BUCKERIDGE, M. S. Sistemas complexos: novas formas de ver a Botânica. Revista Brasil Botânica, v. 27, n. 3, 2004. Disponível em: < http://www.scielo.br/pdf/rbb/v27n3/v27n3a00.pdf>. Acesso em: 7 mar. 2015.

STREIT, R. E.; KLERING, L. R. Governança pública sob a perspectiva dos Sistemas Complexos. In: ENCONTRO DE ADMINISTRAÇÃO PÚBLICA E GOVERNANÇA - EnAPG, 1., Rio de Janeiro, 2004. Anais... Rio de Janeiro: 
ANPAD, 2004. Disponível em: $<$ http://www.anpad.org.br/diversos/trabalhos/EnAPG/enapg 2004/2004 ENAPG227. pdf $>$. Acesso em: 9 ago. 2016.

VAZ, J. C.; RIBEIRO, M. M.; MATHEUS, R. Dados governamentais abertos e seus impactos sobre os conceitos e práticas de transparência no Brasil. Cadernos PPG-AU/FAUFBA, v. 9, n. 1, 2010. Disponível em: $<$ http://www.portalseer.ufba.br/index.php/ppgau/article/view/5111>. Acesso em: 20 fev. 2015.

WAGENSBERG, J. La rebelión de las formas: o cómo perseverar cuando la incertidumbre aprieta. Barcelona: Tusquets, 2004.

WARREN, K.; FRANKLIN, C.; STREETER, C. New directions in systems theory: chaos and complexity. National Association of Social Working, $\mathrm{v}$. 43, n. 4, 1998.4 Disponível em: $<$ http://sw.oxfordjournals.org/content/43/4/357.full.pdf $>$. Acesso em 27 jan. 2015.

WORLD WIDE WEB CONSORTIUM (W3C). SPARQL 1.1 Overview. Massachusetts: W3C/MIT, 2013. Disponível em: <http://www.w3.org/TR/2013/REC-Sparql11-overview-20130321/>. Acesso em: 11 abr. 2014. 